Table 1. Duration of Antimicrobials Among Patients Who Did and Did Not Change Their Code Status

\begin{tabular}{lccc}
\hline Variable & $\begin{array}{c}\text { Patients Not } \\
\text { Changing Code } \\
\text { Status }(\mathrm{n}=96)\end{array}$ & $\begin{array}{c}\text { Patients } \\
\text { ChangingCode } \\
\text { Status }(\mathrm{n}=36)\end{array}$ & $\begin{array}{c}P \\
\text { Value }\end{array}$ \\
\hline $\begin{array}{c}\text { Discharged on } \\
\text { antimicrobials, no. (\%) }\end{array}$ & $36(37.5)$ & $6(16.7)$ & .022 \\
\hline $\begin{array}{c}\text { Total duration (days) of } \\
\text { antimicrobials (inpatient }+ \\
\text { outpatient), median (IQR) }\end{array}$ & $9(4.3-22.3)$ & $6.5(4-12)$ & .044 \\
\hline $\begin{array}{c}\text { Duration (days) of inpatient } \\
\text { antimicrobials, median (IQR) }\end{array}$ & $6(4-12.8)$ & $5(4-9.5)$ & NS \\
\hline
\end{tabular}

Note. IQR, interquartile range; NS, not significant.

interquartile range [IQR], 3-7) than patients not discharged to hospice ( $\mathrm{n}=109$; median, 7 days; IQR, 4-12). When analyzing by randomization arm, we found no statistically significant difference in discharge on antimicrobial therapy $(P=.14)$. However, patients in the control arm of the study were assigned to usual care, in which they could undergo palliative-care consultation.

\section{Discussion}

In a cohort of patients enrolled in a randomized controlled trial of early palliative-care consultation, patients who changed their code status were less likely to be discharged on antimicrobials and to receive overall shorter courses of antimicrobials. This difference was primarily driven by patients transitioning to hospice $(n=23)$ with 0 of 23 patients discharged on antimicrobials, a number substantially lower than in previous studies. ${ }^{4}$

Palliative care consultants help clarify resuscitation preferences and discuss the risks and benefits of many different therapies for patients at the end of life; antimicrobials are among these. Antimicrobials in patients at the end of life are frequently used but with uncertain benefit. ${ }^{5,7}$ Our study suggests that early palliative-care consultation, even when not designed as an antimicrobial stewardship intervention, may nonetheless be effective in reducing antimicrobial consumption in patients at the end of life. Antimicrobial stewardship programs should consider engaging palliative-care providers in the development of endof-life antimicrobial stewardship efforts.

Author ORCIDs. Jason Burnham, (iD) 0000-0002-4777-3006

\section{Acknowledgments.}

Financial support. Dr. Kollef was supported by the Barnes-Jewish Hospital Foundation. This publication was made possible by the NIH-National Center for Advancing Translational Sciences (NCATS), components of the National Institutes of Health (NIH), and NIH Roadmap for Medical Research (grant no. UL1 TR002345, subaward KL2 TR002346). Its contents are solely the responsibility of the authors and do not necessarily represent the official view of NCATS or NIH.

Conflicts of interest. All authors have no conflicts of interest to report.

\section{References}

1. Vincent JL, Rello J, Marshall J, et al. International study of the prevalence and outcomes of infection in intensive care units. JAMA 2009;302: 2323-2329.

2. Antibiotic resistance threats in the United States, 2013. Centers for Disease Control and Prevention website. https://www.cdc.gov/drugresistance/pdf/arthreats-2013-508.pdf. Published 2013. Accessed February 12, 2019.

3. Thorpe KE, Joski P, Johnston KJ. Antibiotic-resistant infection treatment costs have doubled since 2002, now exceeding $\$ 2$ billion annually. Health Affairs 2018;37:662-669.

4. Thompson AJ, Silveira MJ, Vitale CA, Malani PN. Antimicrobial use at the end of life among hospitalized patients with advanced cancer. Am J Hosp Palliat Care 2012;29:599-603.

5. Reinbolt RE, Shenk AM, White PH, Navari RM. Symptomatic treatment of infections in patients with advanced cancer receiving hospice care. J Pain Symptom Manage 2005;30:175-182.

6. Ma J, Chi S, Buettner B, et al. Early palliative care consultation in the medical ICU: a cluster randomized crossover trial. Crit Care Med 2019 (in review).

7. Rosenberg JH, Albrecht JS, Fromme EK, et al. Antimicrobial use for symptom management in patients receiving hospice and palliative care: a systematic review. J Palliat Med 2013;16:1568-1574.

\title{
A major monoclonal hospital outbreak of NDM-1-producing Klebsiella pneumoniae ST340 and the first report of ST2570 in Brazil
}

\author{
Jussimara Monteiro ${ }^{1}$ (1), Fernanda M. Inoue ${ }^{1}$, Ana Paula T. Lobo ${ }^{2}$, Aline S. Ibanes ${ }^{3}$, Sergio Tufik ${ }^{1}$ and Carlos R. V. Kiffer ${ }^{4}$ \\ ${ }^{1}$ Department of Research and Development, Associação Fundo de Incentivo a Pesquisa (AFIP - Medicina Diagnóstica), São Paulo, Brazil, ${ }^{2}$ Clinical Microbiology \\ Section, Associação Fundo de Incentivo a Pesquisa (AFIP - Medicina Diagnóstica), São Paulo, Brazil, ${ }^{3}$ Infection Control Service, Complexo Hospitalar Municipal \\ de São Caetano do Sul, São Paulo, Brazil and ${ }^{4}$ Division of Infectious Diseases, Department of Internal Medicine, Universidade Federal de São Paulo, Escola \\ Paulista de Medicina, São Paulo, Brazil
}

Author for correspondence: Jussimara Monteiro, Email: jussimara.nurmberger@afip. com.br

PREVIOUS PRESENTATION: This report was presented in part at session 229 as poster no. 487 at the ASM MICROBE conference on May 9, 2018, in Atlanta, Georgia.

Cite this article: Monteiro J, et al. (2019). A major monoclonal hospital outbreak of NDM-1-producing Klebsiella pneumoniae ST340 and the first report of ST2570 in Brazil. Infection Control \& Hospital Epidemiology, 40: 492-494, https://doi.org/10.1017/ice.2018.333
To the Editor-New Delhi metallo- $\beta$-lactamase (NDM) is one of the main globally described carbapenemases. It was first reported in 2009 in India. ${ }^{1}$ Providencia rettgeri was first reported in Brazil in 2013. ${ }^{2} \mathrm{NDM}$ emergence has been described in Brazil among gramnegative bacteria related to infection or the environment. ${ }^{3-5}$ Here, we describe an outbreak of NDM-1-producing Klebsiella pneumoniae (KPN) strains ST340 and ST2570 in 50 single isolates from 2 Brazilian hospitals between May 2017 and July 2018 (Figure 1). 
Distribution along time of NDM KPN hospital infection (HI) cases and colonizations (Col)

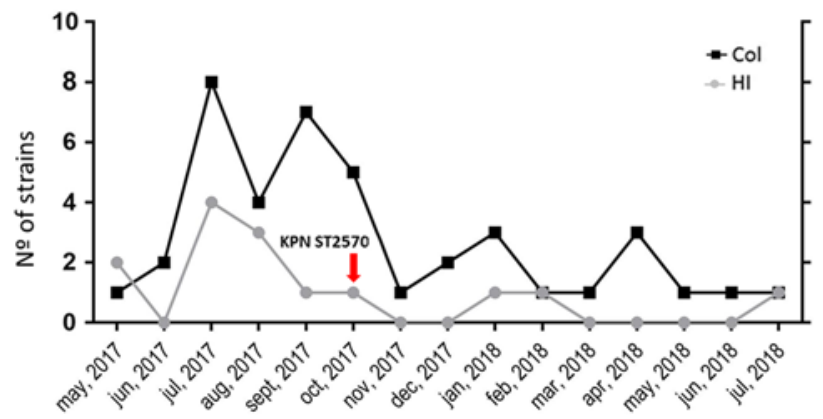

Fig. 1. New Delhi metallo- $\beta$-lactamase producing Klebsiella pneumoniae (NDM KPN) case distribution along time.

These hospitals belong to a regional medical complex with different locations and professionals. Their microbiology laboratory has applied the same metallo- $\beta$-lactamase $(\mathrm{M} \beta \mathrm{L})$ screening procedure with commercial carbapenem disks with and without EDTA (0.1 M) since 2016. Patients admitted are routinely screened for the presence of multidrug-resistant Enterobacteriaceae. The lab identified $50 \mathrm{KPN}-\mathrm{M} \beta \mathrm{L}$-positive carbapenem-resistant single strains using the Vitek MS system (bioMeriéux, Marcy-I'Étoile, France). One sample per patient was considered, and the $b l a_{\mathrm{NDM}-1}$ gene was detected using multiplex real-time polymerase chain reaction (qPCR). ${ }^{6}$

Overall, 45 isolates were identified from hospital 1, and 5 were identified from hospital 2. Among them, 41 were from surveillance cultures (rectal swabs) and 9 were from clinical samples (5 urine, 3 blood and 1 tracheobronchial aspirate). At the moment of isolation, 14 of these 50 patients had a diagnosis of hospital-acquired infection (HAI) according to established criteria (7 nonventilation pneumonia, 4 bloodstream, 2 catheter, and 1 skin and soft-tissue infections). In 5 of 14 patients, NDM-KPN was potentially associated with the HAI ( 3 blood and 2 urine cultures). In 5 of the $14 \mathrm{HAI}$ patients, other microorganisms were considered causative agents, and in 4 these patients, no agents were isolated from infections (NDM KPN from surveillance only). At admission, 7 of the 14 HAI patients presented with community infections, 4 with orthopedic or trauma, and 2 with neurological conditions. Also, 5 of these $14 \mathrm{HAI}$ patients had multiple comorbidities. Notably, 7 of these 14 patients were later discharged with improved conditions, and 7 of these patients died during their hospital stay.

Antimicrobial susceptibility testing was performed by Vitek 2 System (bioMeriéux), and high-level resistance was detected for all $\beta$-lactams, ciprofloxacin, and gentamicin (ie, using the guidelines of the Clinical Laboratory Standards Institute [CLSI]). All strains were susceptible to colistin (minimum inhibitory concentration [MIC], $\leq 2 \mu \mathrm{g} / \mathrm{mL}$ ), and 40 of 50 were resistant to tigecycline (MIC, $>2 \mu \mathrm{g} / \mathrm{mL}$ ) (ie, using the European Committee on Antimicrobial Susceptibility Testing (EUCAST) criteria). ES $\beta$ Ls (ie, $b l a_{\mathrm{TEM}}, b l a_{\mathrm{SHV}}$, and $b l a_{\mathrm{CTX}-\mathrm{M}}$ ) and quinolone resistance genes ( $q n r S)$ were detected in all isolates. A molecular investigation was performed using pulsed field gel-electrophoresis (PFGE), and multilocus sequence typing (MLST). PFGE showed 2 different patterns, A and B. Pattern A was found in 44 of 45 strains from hospital 1 and in 5 of 5 strains from hospital 2. Only 1 strain from hospital 1 showed a distinct PFGE pattern (pattern B). MLST was performed in $5 \mathrm{KPN}$ strains, 4 belonging to pattern A and 1 belonging to pattern B. Also, 4 pattern A strains belonged to the same ST340, clonal complex 258 (CC258). The pattern B strain belonged to ST2570, a different and nonphylogenetically related strain.
The first (or index) case occurred in hospital 1 in May 2017 on a female patient hospitalized at an isolation unit. This patient had many comorbidities (ie, diabetes, hypertension, Alzheimer disease, and previous hemorrhagic stroke) and was admitted to the hospital due to urinary tract infection. On May 21, 2017, she was diagnosed with nonventilation pneumonia and presented both KPN and Enterobacter cloacae from blood cultures. Despite therapy, she died during hospitalization on July 1, 2017. The KPN isolate belonged to PFGE pattern A and ST340.

Various studies have demonstrated that the KPN phylogenetic lineage belonging to CC258 (including ST258, ST11, ST340, and ST437) is vastly adapted to human populations and to hospital infections. ${ }^{7}$ Previous reports of KPN ST340 and ST11 carrying NDM and other resistance mechanisms (ES $\beta$ Ls and $q n r$ genes) have been documented in Brazil. ${ }^{4,5}$ The emergence and spread of NDM KPN is worrying and represents a new worldwide challenge because it may carry several high-level antimicrobial resistance mechanisms.

The KPN pattern B and ST2570 strain was recovered from a blood culture of a patient hospitalized on a different unit in hospital 1 on October 6, 2017. This patient was initially hospitalized on September 20, 2017, due to a skin and soft-tissue infection and various comorbidities (ie, human immunodeficiency virus [HIV], treated tuberculosis, and chronic obstructive pulmonary disease [COPD]) and with many previous hospital passages. The blood culture was positive for NDM KPN on October 6, 2017, and the HAI was a skin and soft-tissue infection. Unfortunately, this patient was sent to the intensive care unit (ICU) and died 15 days later, despite medical care and antimicrobial therapy (including meropenem and polymixin B). Notably, the detection of the KPN ST2570 isolate seems relevant. Eibach et $\mathrm{al}^{8}$ described ES $\beta$ L-producing KPN ST2570 in samples from local and imported poultry in Ghana. To the best of our knowledge, this is the first human case (bloodstream infection and sepsis) caused by an NDM-KPN ST2570. It has been shown that NDM plasmid-bearing microorganisms might have significant environmental presence, ${ }^{9,10}$ which could be gene sources to other species. We do not know which role the ST2570 isolate played in this outbreak, but it presence raises an interesting discussion, especially in a patient with multiple comorbidities and admittances. Could distinct KPN lineages adapted to poultry be the linkage between CC258 and environmental NDM isolates?

We report the first major outbreak of NDM KPN in hospitals from a single city in Brazil. Most isolates belonged to a well-adapted and documented CC258 (ST340). One case was caused by a distinct and not phylogenetically related ST2570. This outbreak highlights the relevance of monoclonal isolate dissemination between environments, despite hospital control policies. Apparently, monoclonal dissemination of well-adapted isolates takes place before plasmid dissemination to other clones or species.

Author ORCIDs. Jussimara Monteiro (iD 0000-0002-1230-4816

Financial support. No financial support was provided relevant to this article.

Conflicts of interest. All authors report no conflicts of interest relevant to this article.

\section{References}

1. Yong D, Toleman MA, Giske CG, Cho HS, Sundman K, Lee K, Walsh TR. Characterization of a new metallo-beta-lactamase gene, bla (NDM-1), and a novel erythromycin esterase gene carried on a unique genetic structure in Klebsiella pneumoniae sequence type 14 from India. Antimicrob Agents Chemother 2009;53:5046-5054. 
2. Carvalho-Assef AP, Pereira PS, Albano RM, et al. Isolation of NDM-producing Providencia rettgeri in Brazil. J Antimicrob Chemother 2013;68:2956-2957.

3. Rozales FP, Magagnin CM, Campos JC, et al. Characterization of transformants obtained from NDM-1-producing Enterobacteriaceae in Brazil. Infect Control Hosp Epidemiol 2017;38:634-636.

4. Aires CA, Pereira PS, de Araujo CF, et al. Multiclonal expansion of Klebsiella pneumoniae isolates producing NDM-1 in Rio de Janeiro, Brazil. Antimicrob Agent Chemother 2017;61:e01048-16.

5. Campana EH, Montezzi LF, Paschoal RP, Picão RC. NDM-producing Klebsiella pneumoniae ST11 goes to the beach. Int J Antimicrob Agent 2017;49:119-121.

6. Monteiro J, Widen RH, Pignatari AC, Kubasek C, Silbert S. Rapid detection of carbapenemase genes by multiplex real-time PCR. I Antimicrob Chemother 2012;67:906-909.
7. Castanheira M, Costello AJ, Deshpande LM, Jones RN. Expansion of clonal complex 258 KPC-2-producing Klebsiella pneumoniae in Latin American hospitals: report of the SENTRY Antimicrobial Surveillance Program. Antimicrob Agent Chemother 2012;56:1668-1669.

8. Eibach D, Dekker D, Gyau Boahen K, et al. Extended-spectrum beta-lactamase-producing Escherichia coli and Klebsiella pneumoniae in local and imported poultry meat in Ghana. Vet Microbiol 2018;217:7-12.

9. Nordmann P, Poirel L, Walsh TR, Livermore DM. The emerging NDM carbapenemases. Trends Microbiol 2011;19:588-595.

10. Walsh TR, Weeks J, Livermore DM, Toleman MA. Dissemination of NDM1 positive bacteria in the New Delhi environment and its implications for human health: an environmental point prevalence study. Lancet Infect Dis 2011;11:355-362.

\title{
Putative horizontal transfer of carbapenem resistance between Klebsiella pneumoniae and Kluyvera ascorbata during abdominal infection: A case report
}

\author{
Otávio Hallal Ferreira Raro MSc ${ }^{1}$ (1), Daiana de Lima-Morales $\mathrm{PhD}^{2}$, Afonso Luis Barth $\mathrm{PhD}^{2}$, Tiago Galvão Paim $\mathrm{PhD}^{3}$, \\ Mariana Preussler Mott MSc ${ }^{1}$, Cezar Vinícius Würdig Riche MSc ${ }^{1}$, Uirá Fernandes Teixeira PhD ${ }^{4}$, Fábio Luiz Waechter PhD \\ and Cícero Armídio Gomes Dias PhD ${ }^{1}$ \\ ${ }^{1}$ Departamento de Ciências da saúde da Universidade Federal de Ciências da Saúde de Porto Alegre - UFCSPA, Porto Alegre, Rio Grande do Sul, Brazil, \\ ${ }^{2}$ LABRESIS - Laboratório de Pesquisa em Resistência Bacteriana, Hospital de Clínicas de Porto Alegre, Porto Alegre, Rio Grande do Sul, Brazil, ${ }^{3}$ Instituto de \\ Biociências da Universidade Federal do Rio Grande do Sul - UFRGS, Porto Alegre, Rio Grande do Sul, Brazil, Brazil and ${ }^{4}$ Serviço de Cirurgia do Aparelho \\ Digestivo da UFCSPA/Santa Casa - UFCSPA, Porto Alegre, Rio Grande do Sul, Brazil
}

To the Editor-The emergence of KPC-producing Enterobacter has led to the development of serious infections related to high levels of mortality and morbidity worldwide. ${ }^{1,2}$ The rapid spread of KPCs is linked to multiple elements, such as plasmid-borne genes and the dissemination by international travelers; these bacteria are frequently multidrug resistant, causing untreatable infections. ${ }^{3,4}$ Kluyvera spp is a genus of gram-negative rods of the Enterobacteriaceae family. ${ }^{5}$ Although it is a commensal of the human gut microbiota, ${ }^{6}$ Kluyvera spp has the potential to cause septic shock, urinary tract infections, catheter-associated bloodstream infections, and abdominal infections. ${ }^{7}$ Here, we report a case of a plasmid-mediated horizontal transfer from a Klebsiella pneumoniae isolate to a Kluyvera ascorbata isolate during abdominal infection. The patient approved the data submission.

A 43-year-old male patient was admitted to the Hepatobiliary and Pancreatic Surgical Division from a hospital in the South Region of Brazil in October 2016. He was asymptomatic but had an incidental type 1 biliary cyst that was discovered during ultrasonography. Magnetic resonance imaging (MRI) with cholangiopancreatography was performed for adequate evaluation and showed an abnormal pancreatobiliary junction, as well. In November 2016, the patient underwent a cholecystectomy and total resection of the cyst, with closure of distal part of the main bile duct inside the pancreas, accompanied by Roux-en-Y hepaticojejunostomy to provide proper

\footnotetext{
Author for correspondence: Otávio Hallal Ferreira Raro, E-mail: otaviohraro@gmail. com

Cite this article: Raro OHF, et al. (2019). Putative horizontal transfer of carbapenem resistance between Klebsiella pneumoniae and Kluyvera ascorbata during abdominal infection: A case report. Infection Control \& Hospital Epidemiology, 40: 494-496, https:// doi.org/10.1017/ice.2019.26
}

biliary drainage. The pathology report showed no malignancy in surgical specimen. After 48 hours, the patient was evaluated with postoperative pancreatitis and signs of sepsis, therefore piperacillin/ tazobactam treatment was started. The patient continued to present clinical deterioration and needed parenteral nutrition; he was consequently transferred to the intensive care unit (ICU). Blood cultures were negative and abdominal computerized tomography (CT) showed abdominal collections. CT-guided drainage of pancreatic fluid was performed and cultures were negative. Nevertheless, antimicrobial treatment with meropenem was started and continued for 14 days without resolution. A second CT-guided drainage procedure was performed at the end of December, and the bacteriological culture yielded a multisusceptible Enterococcus faecalis; a Kluyvera ascorbata resistant to ampicillin and second-generation cephalosporin but susceptible to carbapenem, and multidrugresistant Klebsiella pneumoniae, including resistance to tigecycline and carbapenem. Antibiotics were adjusted to vancomycin, meropenem, and ertapenem plus polymyxin B with clinical improvement but without complete bacterial clearance. After 17 days, a third $\mathrm{CT}$-guided drainage was performed, and the bacterial culture yielded $1 \mathrm{~K}$. ascorbata isolate resistant to carbapenem. A fourth CT-guided drainage was performed after 15 days, and the culture yielded $K$. ascorbata susceptible to carbapenem and K. pneumoniae resistant to carbapenem. Antibiotic treatment was adjusted to polymyxin B, tigecycline, and sulfametoxazol-trimetoprim, and the patient was evaluated with signs of controlled infection. After 14 days, the antibiotic treatment was suspended, and a final CT showed no signs of abdominal collections. The patient was discharged and was followed as an outpatient. 